\title{
Avaliação de impacto do Programa Inverno Gaúcho no estado do Rio Grande do Sul
}

Marília Patta Ramos ${ }^{1}$

Graça Godinho

1 Ph.D em Sociologia. Professora da Universidade Federal Rio Grande do Sul (UFRGS).

2 Doutoranda em Economia pela Universidade Federal do Rio Grande do Sul (UFRGS). 


\section{Resumo}

ESTE TRABALHO É UMA EXPERIÊNCIA DE AVALIAÇÃO DE

IMPACTO do Programa INVERNo GaÚCHO (ProIG), DA

ÁREA DA SAÚDE, NO ESTADO DO RIO GRANDE

DO SUL. OS PRINCIPAIS OBJETIVOS FORAM VERIFICAR O EFEITO DO PROGRAMA NO NÚMERO DE INTERNAÇÕES POR INFECÇÃO RESPIRATÓRIA AGUDA (IRA) ENTRE ADULTOS COM 60 ANOS OU MAIS (IDOSOS), EM DIFERENTES REGIÕES DO RIO GRANDE DO SUL, ENTRE OS ANOS DE 2005 E 2010. A PARTIR DA ORGANIZAÇÃO DOS DADOS, FORAM OBSERVADAS ALGUMAS DIMENSÕES, COMO A INVIABILIDADE DA COMPARAÇÃO ENTRE ALGUNS MUNICÍPIOS, VISTO OQUE SUAS CARACTERÍSTICAS SÃO INCOMPATÍVEIS, APONTANDO A NECESSIDADE DE UM PAREAMENTO ANTES DAS ANÁLISES. REALIZOU-SE O PAREAMENTO DOS MUNICÍPIOS POR MEIO DA TÉCNICA DE PROPENSITY SCORE MATCHING, A FIM DE EOUALIZAR AS DIFERENÇAS ENTRE AMOSTRAS. OBSERVOU-SE DIFERENÇA ESTATISTICAMENTE SIGNIFICATIVA SOMENTE NO ANO DE 2007, NO SENTIDO DE OUE OS MUNICÍPIOS OUE ADERIRAM AO ProIG APRESENTARAM TAXA MENOR QUE AOUUELES OUUE NÃO ADERIRAM.

\section{Abstract}

THIS ARTICLE REFERS TO AN EXPERIENCE RELATED TO A POLICY EVALUATION. SPECIFICALLY IT IS PRESENTED THE EVALUATION OF A PROJECT DEVELOPED BY RIO GRANDE Health Secretary: the InVERno Gaucho Project, WHICH INTENDS TO REDUCE THE HOSPITALIZATION AMONG ADULTS $6 O$ YEARS OR OLDER (ELDERLY) DUE TO RESPIRATORY INFECTION. THIS EVALUATION WAS DONE WITH CITIES THAT PARTICIPATED IN THE PROJECT IN COMPARISON TO THOSE CITIES THAT DIDN'T PARTICIPATE. THE USE OF A COMPARATIVE GROUP OF COUNTIES THAT DIDN'T PARTICIPATE WAS NECESSARY TO CONTROL FOR CHARACTERISTICS THAT CAN AFFECT THE HOSPITALIZATION DUE TO RESPIRATORY INFECTION RATES (DEPENDENT VARIABLE) THAT ARE NOT RELATED TO THE PROJECT. IN TERMS TO THE ANALYTICAL PROCEDURES DESCRIPTIVE ANALYSIS WERE DONE TO COMPARE MEANS AND ALSO MULTIVARIATE REGRESSION MODELS WERE RUN TO ESTIMATE THE DEPENDENT VARIABLE. IT WAS OBSERVED THAT THE GREATEST MEAN DIFFERENCE AMONG PARTICIPANTS AND NON PARTICIPANTS HAPPENED DURING THE YEAR 2007. AND, THIS DIFFERENCE POINTED THAT PARTICIPANTS PRESENTED LOWER HOSPITALIZATION DUE TO RESPIRATORY INFECTION THAN THOSE NON PARTICIPANTS.

\section{PALAVRAS-CHAVE:}

Políticas públicas; Avaliação de impacto; Programa Inverno Gaúcho.

Revista Brasileira de Monitoramento e Avaliação | Número 9 | Janeiro-Junho de 2015 


\section{Introdução}

As pesquisas de avaliação de impacto de políticas públicas não apresentam um marco teórico específico. Elas são aplicadas, e a teoria que as compõe diz respeito muito mais aos postulados teóricos que informam o desenho de cada política em particular.

O objetivo geral desta pesquisa foi avaliar o impacto do Projeto Inverno Gaúcho (Proig) na redução na taxa de internação por infecção respiratória aguda (IRA) em idosos no Rio Grande do Sul (RS), nos meses de junho, julho e agosto.

Os objetivos específicos foram:

- Identificar, nos municípios que aderiram ao programa, a taxa de internação por infecção respiratória aguda em idosos antes e após a adesão ao programa.

- Comparar a taxa de internação por infecção respiratória aguda em idosos nos municípios-alvo com outros similares, mas que não entraram no programa.

Com relação à avaliação de políticas públicas, destaca-se que vários pesquisadores apontam a sua importância no Brasil, observando-se especialmente a proliferação de pesquisas de avaliação, que podem ser úteis para medir a eficácia da gestão pública e decisivas para o processo de aprendizagem institucional. A avaliação contribui para a busca e obtenção de ganhos das ações governamentais em termos de satisfação dos usuários e de le- gitimidade social e política. Por varias razões, ressalta-se a relevância dos processos de avaliação para a reforma das políticas públicas, a modernização e a democratização da gestão pública (CANO, 2006).

A avaliação de impacto, conforme Carvalho (2003:186), "[...] é aquela que focaliza os efeitos ou impactos produzidos sobre a sociedade e, portanto, para além dos beneficiários diretos da intervenção pública, avaliando-se sua efetividade social". Busca-se detectar as mudanças que efetivamente ocorreram e em que medida essas transformações se deram na direção desejada. De acordo com a autora, dois pressupostos orientam a avaliação de impacto: o primeiro reconhece propósitos de mudança social na política em análise, e, dessa forma, faz sentido configurar a investigação para mensurar seus impactos; o segundo estrutura a análise com base em uma relação causal entre o programa e a alteração social provocada. Não basta constatar a ocorrência de mudança, é preciso estabelecer a relação causa-efeito entre as ações de um programa e o resultado final obtido e entre essas e a ação pública realizada por meio da política (GARCIA, 2001; CARVALHO, 2003; COSTA e CASTANHAR, 2003).

A avaliação é uma função de gestão destinada a auxiliar o processo de decisão, visando torná-lo o mais racional e efetivo possível. Ela pode subsidiar o planejamento e a formulação das intervenções governamentais, 
o acompanhamento de sua implementação, suas reformulações e ajustes, assim como as decisões sobre a manutenção ou a interrupção das ações. É um instrumento importante para a melhoria da eficiência do gasto público, da qualidade da gestão e do controle sobre a efetividade da ação do Estado, bem como para a divulgação de resultados de governo (GARCIA, 2001).

Em termos técnicos, conforme Ramos (2009), quando se pensa em avaliação de impacto de uma política, deve-se ter informações sobre as condições do público-alvo, como as regiões, instituições ou qualquer outra singularidade que sirva de análise, antes da política ter sido implantada. Sem essas informações, torna-se praticamente inviável qualquer tipo de avaliação de impacto.

Quanto à estrutura deste artigo, na primeira seção, faz-se uma descrição referente ao contexto do projeto e sua avaliação; na segunda, expõe-se a metodologia utilizada para avaliar o impacto; na terceira, discutem-se os resultados da avaliação; e, por fim, são feitas as considerações finais relativas ao projeto e às questões mais amplas que surgiram desta experiência.

\section{Contextualização e avaliação do Projeto Inverno Gaúcho}

O Governo do Estado do Rio Grande do Sul, por meio da Secretaria da Saúde, desenvolveu o Projeto Inverno Gaúcho a partir de 2008. 0 projeto teve por objetivo controlar as infecções respiratórias agudas (IRA), responsáveis por um número elevado de internações e óbitos de pessoas com 60 anos e mais no estado.

Dentre as atividades propostas, estava a capacitação dos profissionais de saúde dos municípios, além da ampliação do acesso da população aos serviços de saúde nos meses de maior intensidade do inverno (junho a agosto).

As ações consistiam em: a) repasse de recursos financeiros para a abertura de um terceiro turno de atendimento em postos de saúde (até as 22 horas); b) fornecimento de kits de medicamentos nos dois primeiros anos e, depois, a relação dos medicamentos recomendados; c) capacitação de técnicos das coordenadorias regionais de saúde responsáveis pela multiplicação das informações das normas técnicas e operacionais³ (NTO) para a rede básica municipal; e d) ações de educação com foco nas doenças de inverno por meio dos meios de comunicação.

3 A finalidade da norma técnica e operacional é fornecer subsídios para o atendimento correto das infecções respiratórias agudas (IRA) na rede de serviços do Sistema Único de Saúde no estado do Rio Grande do Sul. (Norma técnica e operacional para infecções respiratórias agudas (IRA) em pessoas com 60 anos e mais. $3^{a}$ edição - atualizada - 2008).

Revista Brasileira de Monitoramento e Avaliação | Número 9 | Janeiro-Junho de 2015 
Além dessas ações, seriam realizados repasses mensais de recursos, durante os meses de inverno, aos municípios que aderissem espontaneamente ao projeto. Cada município teria capacitação e deveria implantar o projeto, recebendo os repasses de recursos para a efetivação das ações.

A avaliação de uma política ou projeto parte do pressuposto geral de que existe um âmbito de ação coletiva possível de ser delimitado, que envolve governo, sociedade e mercado em uma complexidade de relações, interações e movimentos. Essa dinâmica resulta em uma ou mais decisões a respeito de um tema social relevante, a serem consolidadas em lei, ordenando diretrizes, ações, recursos, objetivos e metas, de forma permanente e organizada pelo poder público. Isso pode ser qualificado de âmbito das "políticas públicas" (FERREIRA, 2007). Peters (1986) apud Souza (2006) aborda política pública como a soma das atividades dos governos que influenciam a vida dos cidadãos.

A avaliação foi realizada em uma situação na qual o Proig não possuía outras metas definidas ou indicadores além das taxas de internação.

Desse modo, as questões norteadoras foram:

1. Qual o impacto do Projeto Inverno Gaúcho sobre o número de internações por IRA em idosos nos diferentes municípios do Rio Grande do Sul?

2. Existem diferenças entre taxas de internação de municípios com caracte- rísticas similares que fazem parte do referido projeto e dos que não fazem?

A partir dessas questões, foram definidos os objetivos do presente trabalho, que eram avaliar o impacto do Projeto Inverno Gaúcho na redução na taxa de internação por infecção respiratória aguda (IRA) em idosos no RS nos meses de atuação do projeto.

Em seguida, coube:

- Identificar, nos municípios que aderiram ao projeto, a taxa de internação por infecção respiratória aguda (IRA) em idosos antes e após a adesão ao projeto.

- Comparar a taxa de internação por infecção respiratória aguda (IRA) em idosos nos municípios-alvo com a de outros municípios similares, mas que não aderiram ao projeto.

\section{Metodologia}

Foi realizada uma pesquisa de dados primários, por meio de contato telefônico com as secretarias de Saúde de todos os municípios do RS, questionando sobre a participação ou não no Projeto Inverno Gaúcho.

O projeto analisado possui uma grande disponibilidade de informações e uma avaliação de seu impacto, realizada em 2008 por integrantes da Secretaria da Saúde do Rio Grande do Sul. No entanto, não havia uma análise que levasse em conta a diferença entre municípios que aderiram e os que não participaram do Proig, nos períodos anterior e posterior à sua implantação. 
A avaliação aqui apresentada tomou um grupo comparativo não atingido pela política, para evitar vieses como as características peculiares do grupo atingido ao longo do tempo, que podem mascarar ou até mesmo exacerbar o efeito da política que está sendo apreciada. Assim, considera-se essa avaliação válida, na medida em que segue critérios científicos.

Especificamente, quando se tenta verificar o impacto de uma política/programa social neste caso, o Proig -, lida-se com relações de causa e efeito. O que se pretende é identificar o efeito desse programa/política em alguma dimensão da vida econômica, social ou política de uma determinada sociedade Sendo assim, a lógica por trás das pesquisas de avaliação está inserida na lógica dos experimentos.

A partir da organização completa dos dados, foram observadas algumas dimensões, como a impossibilidade de comparação entre alguns municípios, visto que suas características eram incompatíveis. Esse fato apontou a necessidade de um pareamento. Tal técnica consiste em identificar que as unidades não tratadas (municípios que não aderiram ao Proig) são similares às tratadas (municípios que aderiram ao Proig) e comparar as médias no resultado (médias de internações por IRA após o Proig) procurado entre esses dois grupos para identificar o impacto do tratamento (projeto). Os procedimentos de pareamento são implementados utilizando-se um escore balanceado, computado a partir de um modelo de regressão logística no qual a variável dependente seria 1 = aderiu e 0 = não aderiu.

Especificamente, usou-se o logaritmo das chances de aderir ao Proig (log odds). Estimou-se um modelo logit utilizando-se todas as variáveis observadas como preditoras, para se obter a probabilidade predita e computar a razão das chances (log odds-ratio) para cada observação na amostra do grupo controle (não aderiu ao Proig) e do grupo tratamento (aderiu ao Proig).

Basicamente, o procedimento dessa técnica testa se os escores de propensão a aderir, tendo como variáveis independentes algumas características observadas, são estatisticamente os mesmos nos grupos controle e tratamento. Se não forem, o processo continua, com a retirada de unidades que estão mais distantes, até que se tenha um balanço nas observações ${ }^{4}$. Uma vez que haja um certo balanço, isto é, que as unidades estejam comparáveis, pode-se efetuar o teste de diferença de médias da variável que se refere ao

4 Tal técnica nem sempre é eficiente, posto que, em situações com poucos casos, ela torna a amostra menor ainda por retirar casos não tratados que não sejam comparáveis aos casos tratados. Assim, nem sempre o uso dessa técnica é viável. Para maiores esclarecimentos, ver FOSTER, Michael Propensity Score Matching: An illustrative analysis of dose response. Medical Care, vol. 41, nr 10, pp 1183-1192, (2003)

Revista Brasileira de Monitoramento e Avaliação | Número 9 | Janeiro-Junho de 2015 
resultado do projeto que se pretende estimar, entre os grupos controle e tratamento. Nesse caso, houve a comparação entre as médias do número de internações por IRA no grupo que aderiu ao Proig e naquele que não participou do projeto.

\subsection{VARIÁVEIS DE ESTUDO E ESTRATÉGIA ANALÍTICA}

As variáveis do estudo foram obtidas por meio do Datasus, do Feedados 5 e do Instituto Brasileiro de Geografia e Estatística (IBGE) e organizadas, em nível municipal, para os anos anteriores e posteriores ao Proig. Especificamente, os dados foram organizados numa planilha do programa SPSS.

Além de análises descritivas, tais como diferenças de médias, utilizou-se o modelo de regressão multivariada. Nesse modelo, a variável dependente era o resultado do cálculo do número de internações após o Proig menos o número de internações antes do Proig. A variável independente principal era dicotômica, valendo 1 se o município participasse do Proig e 0 se não participasse. Além disso, foi incluído o conjunto das variáveis de controle, como PIB, Idese, cobertura pela Estratégia de Saúde da Família, leitos pelo SUS, taxa de urbanização, IDH-M, cobertura vacinal e temperatura média nos meses de inverno (para os anos de 2004 a 2010), que podem impactar as internações.
Como muitos municípios foram aderindo no decorrer do período (2005 até 2010), foi feita inicialmente uma análise na qual a variável dependente resultou da subtração da taxa de internações por IRA após o primeiro ano do projeto (2005) menos a mesma taxa antes (2004). Basicamente, trabalhou-se com análise da "diferença na diferença ${ }^{6 " .}$ Essa técnica utiliza informações sobre participantes e não participantes, coletadas antes da aplicação da política ou projeto social, comparadas com as mesmas informações coletadas após a aplicação da intervenção (RAMOS, 2009).

Dessa forma, a primeira estratégia analítica foi estimar modelos causais multivariados (com variáveis de controle) para cada ano analisado do programa, em comparação com o ano-base (2004), sendo a principal variável independente a participação no programa, e a dependente, a diferença entre as taxas de internação no ano analisado e as do ano-base (2004, ano sem o programa).

O modelo de regressão múltipla pode ser visualizado abaixo:

$\mathrm{Y}=\beta 0+\beta 1+$ variáveis de controle $+\mathrm{e}$

Onde:

$Y=$ diferença na taxa de internações por IRA entre idosos (ano posterior subtraído do ano anterior) 
$\beta 0=$ constante

$\beta 1$ = aderiu ao Proig $(=1)$

Basicamente, a lógica da técnica da diferença da diferença pode ser vista no esquema abaixo:

$$
\operatorname{Proig}(=1)
$$

(Int IRA2005-Int IRA2004)

$$
\text { Não Proig (=0) }
$$

(Int IRA2005-Int IRA 2004)

Para avaliar o impacto em anos mais recentes, foi usada a mesma estratégia, sempre comparando dois anos: um antes do projeto (2004) e outro após (2005, 2006 e assim sucessivamente).

Para lidar com o fato de que não se contava exatamente com os mesmos municípios de um ano para outro, em função de que novos municípios aderiam, enquanto outros deixavam o projeto, recorreu-se à técnica da diferença da diferença, com um banco de dados em formato longo, no qual a adesão (2005 até 2010) tornou-se uma variável dicotômica. Nesse formato, o modelo mediu valores da taxa de internação por IRA como variável dependente, e o impacto do projeto por uma variável interativa entre aderiu no ano tal=1 e não aderiu no mesmo ano=0, multiplicado pelo ano posterior ao projeto.

O esquema abaixo ilustra a análise:

$Y=X_{1}+X_{2}+X_{3}+X_{4} \ldots+e$.

Onde:

$\mathrm{Y}=$ taxa IRA geral para os anos analisados

$\mathrm{X} 1$ = aderiu $=1$ e não aderiu $=0(200 \ldots)$

$X_{2}=$ ano posterior ao projeto $=1(200 \ldots) x$

ano anterior $=0(200 \ldots)$

X3 = interação $X_{1} * X_{2}$ (impacto do projeto)

$X_{4}=$ variáveis de controle anteriores ao ano

que está sendo avaliado

e $=$ termo de erro da regressão

Destaca-se que foi utilizada essa estratégia quando se comparou o impacto do projeto de um ano para outro, ou seja, comparou-se o ano de 2005 com 2006, o ano de 2006 com 2007, e 2007 com 2008, 2008 com 2009, e 2009 com 2010.

O formato longo de planilha necessário nessa análise pode ser ilustrado pelo quadro a seguir.

5 O Feedados reúne informações de natureza socioeconômica relativas ao Rio Grande do Sul e seus municípios.

6 A estratégia chamada de "diferença na diferença" utiliza dados em painel, aplicados para um conjunto de grupos, nos casos em que alguns desses estão expostos a variáveis causais de interesse e outros não. Um importante componente dessa estratégia é a escolha de variáveis para fazer comparações e responder a questões contrafactuais (ANGRIST; KRUEGER, 1999).

Revista Brasileira de Monitoramento e Avaliação | Número 9 | Janeiro-Junho de 2015 
- ouAdRo 1: REPRESENTAÇÃo da PLANILHA DE DADOS NO FORMATO LONGO

\begin{tabular}{lcccc} 
MUNICÍPIO & ANO & $\begin{array}{c}\text { TAXA } \\
\text { IRA }\end{array}$ & $\begin{array}{c}\text { ANO } \\
\text { APÓS=1 }\end{array}$ & ADERIU=1 \\
\hline 1 & 2006 & 12,0 & 0 & 0 \\
1 & 2007 & 13,0 & 1 & 0 \\
2 & 2006 & 25,0 & 0 & 1 \\
2 & 2007 & 10,0 & 1 & 0 \\
\hline
\end{tabular}

Além dessas análises com a principal variável independente (aderiu ou não), testou-se também o impacto de outras variáveis, as quais não medem especificamente o impacto do projeto, mas sim a intensidade desse impacto.

Isso foi feito incluindo-se nos modelos de regressão para medir o impacto do projeto no último ano para o qual se tinham dados, isto é, 2010, a variável dicotômica houve descontinuidade na adesão $=1$ versus não houve descontinuidade $=0$. Outra forma de medir essa intensidade foi incluindo, em outro modelo, a variável independente tempo de adesão em anos.

Como não foi identificado um impacto significativo do Proig nas internações por IRA entre idosos nos meses de inverno no RS por meio dos modelos de regressão descritos acima, a análise tornou-se mais rigorosa em termos de comparação dos municípios. Isso porque talvez estivessem sendo comparados municípios tão diferentes que não permitissem observar um significativo efeito do projeto. Para tanto, os municípios tornaram-se mais comparáveis através da técnica de escore de propensão para pareamento, a fim de se obter o grupo mais homogêneo possível.

\section{Resultados e discussão}

Pode-se observar, no Gráfico 1, que a adesão não foi contínua e nem parelha. Nota-se que houve, até 2009, um aumento no número de municípios que aderiram (em vermelho no gráfico), sendo que, em 2010, ocorreu uma redução. Já as médias nas taxas de internação de idosos por IRA não apontaram uma tendência de redução, mas, de fato, aumentaram através do tempo, como mostra o Gráfico 2. Contudo, deve-se atentar para a existência de desvios padrão muito altos, o que torna a média uma medida frágil. 


\section{- GRÁFICO 1: FREOUUENNCIA DE ADESÃO DOS MUNICÍPIOS GAÚCHOS AO LONGO DA IMPLEMENTAÇÃO DO PROIG}

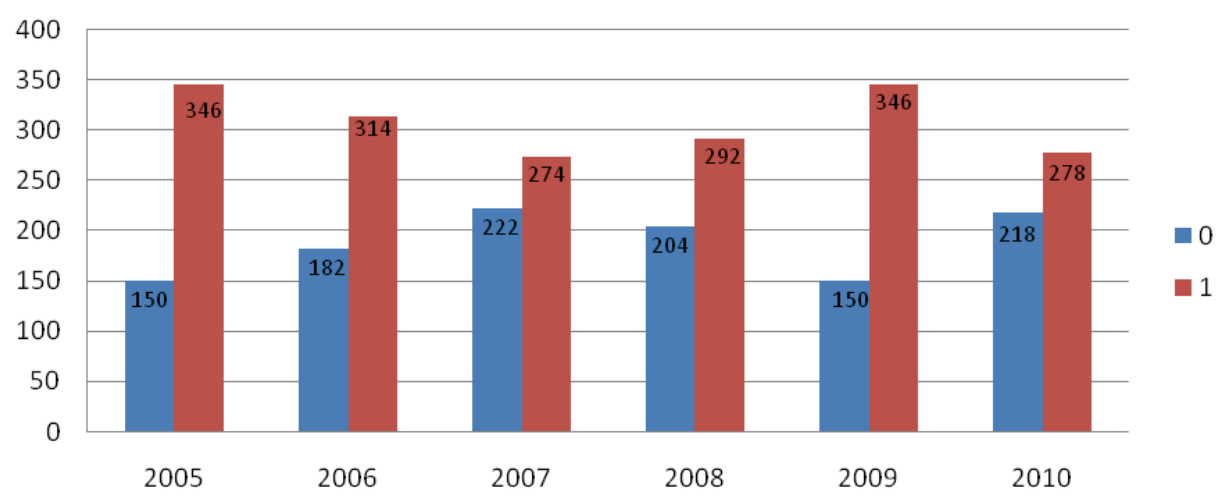

\section{- GRÁFICO 2: FREQ_UÊNCIA DAS TAXAS DE INTERNAÇÃO ENTRE 2004 E 2010}

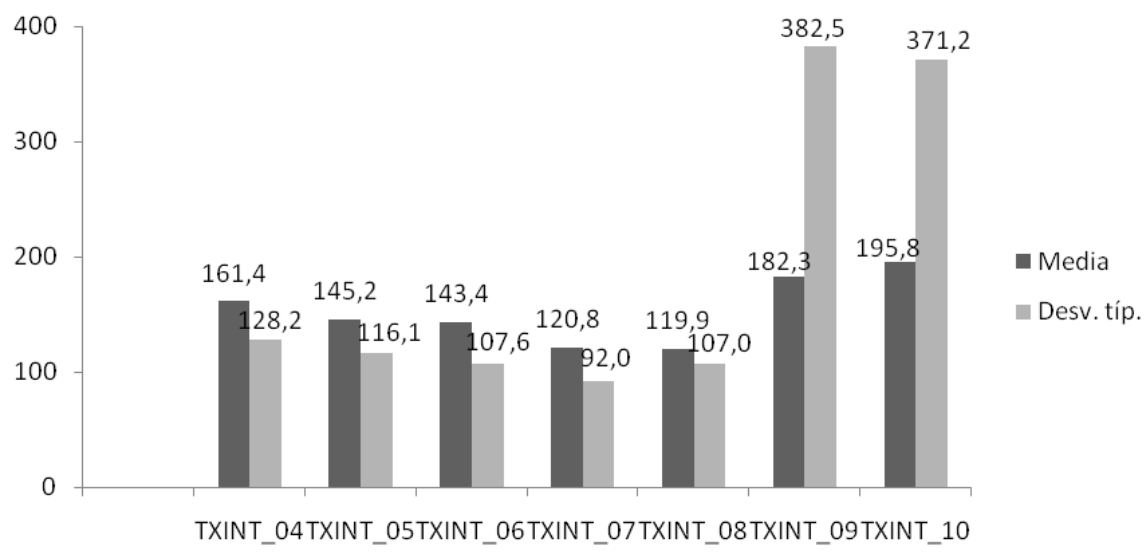

Conforme já mencionado, a pesquisa de avaliação de impacto de programas sociais apresenta dois momentos no tempo (antes e depois de sua implementação) que são essenciais. Sem eles, não haveria como identificar possíveis mu- danças em função do programa que está sendo analisado. Como foi ressaltado anteriormente, deve-se ter dois grupos - neste caso, o que foi atingido pelo Proig (grupo experimental) e o que não foi atingido (grupo de controle). 


\subsection{IMPACTO APÓS PAREAMENTO} COM INDEPENDENTES DO ANO ANTERIOR

Após realizar o pareamento e a diferença nas taxas de internações de idosos por IRA nos meses de inverno no RS para todos os anos (de 2005 até 2010), verificou-se uma diferença estatisticamente significativa somente no ano de 2007. Pode-se visualizar na Tabela 1 que, após o pareamento, os municípios que aderiram ao Proig apresentaram taxa menor do que os que não aderiram.

Com a relação à qualidade do pareamento, verifica-se, pela Tabela 2 abaixo, que somente uma variável, dentre as incluídas na análise os grupos adesão ao Proig x fora do Proig -, apresenta diferença estatisticamente significativa (cobertura vacina gripe) após o pareamento, o que demonstra que ele tornou os grupos equivalentes, evitando o viés de seleção.

- TABELA1: IMPACTO EM 2007 ANTES E APÓS PAREAMENTO (476 CASOS MANTIDOS)

\begin{tabular}{ccccccc} 
VARIÁVEL & AMOSTRA & NO PROIG & FORA DO PROIG & DIFERENÇA & S.E. & T-STAT \\
\hline txintira60_07 & Não pareados & 114.74 & 128.28 & -13.53 & 8.29 & -1.63 \\
& Pareados & 114.58 & 137.80 & -23.22 & 11.35 & -2.05 \\
\hline
\end{tabular}

- TABELA 2: CARACTERÍSTICAS SOCIODEMOGRÁFICAS APÓS O PAREAMENTO ENTRE TRATADOS (NO PROIG) E CONTROLES (FORA DO PROIG)

\begin{tabular}{lccccc}
\multicolumn{1}{c}{ VARIÁVEL } & NO PROIG & FORA DO PROIG & \%BIAS & $T$ & P>|T| \\
\hline ad_07 & 1 & 0 & & & \\
pop_06 & 14728 & 13416 & 1.8 & 0.87 & 0.382 \\
\hline cobpsf_06 & 61.421 & 61.967 & -1.3 & -0.15 & 0.879 \\
\hline pib_06 & 11346 & 11415 & -0.7 & -0.14 & 0.888 \\
\hline idese_06 & .67818 & .6815 & -5.3 & -0.57 & 0.568 \\
hosp_06 & .62992 & .59449 & 2.3 & 0.62 & 0.534 \\
leito_06 & 39.906 & 35.03 & 1.3 & 1.13 & 0.257 \\
taxaurb_06 & 54.889 & 55.843 & -3.7 & -0.40 & 0.687 \\
transsu_06 & 27.624 & 27.7 & -0.7 & -0.09 & 0.929 \\
idhm2000_06 & .74807 & .7493 & -0.6 & -0.09 & 0.930 \\
anosestu_06 & 48.163 & 48.356 & -1.3 & -0.17 & 0.863 \\
rend2000_06 & 224.06 & 222.8 & 1.3 & 0.18 & 0.861 \\
cobertvac_06 & 87.463 & 90.139 & -18.0 & -1.96 & 0.051 \\
tempmédia_06 & 14.436 & 14.547 & -11.2 & -1.25 & 0.212 \\
\hline
\end{tabular}




\section{Considerações finais}

O fato de terem sido encontrados alguns resultados não indicativos de um impacto expressivo do Proig nas taxas de internação levanta alguns questionamentos sobre a maneira como o projeto foi implementado. Basicamente, pensa-se em termos qualitativos, considerando que não basta, para um município, apenas aderir ao projeto, mas é preciso conhecer as condições dessa adesão. Isso passa pelo conhecimento de como o projeto é implementado, se as estratégias estabelecidas pela SES são cumpridas de fato e como são cumpridas. Vários exemplos podem ser citados: a SES aprimorou o acompanhamento, a partir de 2009, no sentido de checar se a expansão no horário de atendimento se realiza de fato.

Considerando que esses aspectos envolvem dimensões mais qualitativas, seria interessante, como agenda futura, pensar numa avaliação de processos (investigar a implementa- ção) ${ }^{7}$, utilizando-se também a supervisão dos municípios e serviços participantes do Proig. Isso pode ajudar a entender os resultados da avaliação aqui apresentada (impacto) ${ }^{8}$, por meio da inclusão de novos indicadores.

A observação da evolução mensal da taxa de internação por IRA em idosos apontou o mês de setembro com índices mais elevados que junho em quatro dos últimos cinco anos. Isso indica a necessidade de incluí-lo nas próximas versões.

Além disso, poderia-se pensar na organização, ou mesmo aprimoramento, do que se tem, ou seja, um banco constantemente alimentado com dados sobre integrantes e não integrantes do Proig. Com isso, se disporia de mais dados ao longo do tempo.

Além dos aspectos já salientados, realizou-se aqui uma avaliação de impacto que não contou com informações teoricamente e nem praticamente selecionadas sobre os municí-

7 Avaliação de processos - realizada durante a implementação do programa, diz respeito à dimensão de gestão. É uma avaliação periódica que procura detectar as dificuldades que ocorrem durante o processo para efetuar correções ou adequações. Serve de suporte para melhorar a eficiência operativa.

8 Avaliação de impactos ou resultados - tem objetivos mais ambiciosos, respondendo se o programa funcionou ou não. Procura verificar em que medida o programa alcança seus objetivos e quais são os seus efeitos, indagando se houve modificações na situação-problema que originou a formulação do programa após sua implementação. Serve de suporte para decisão sobre política, como continuação do programa e formulação de outros.

Revista Brasileira de Monitoramento e Avaliação | Número 9 | Janeiro-Junho de 2015 
pios no momento anterior ao Proig9. Tiveram que ser utilizados dados secundários dos censos do IBGE, muitas vezes defasados.

O ideal seria que, antes da implementação do Proig ${ }^{10}$, fosse feita uma pesquisa de diagnóstico sobre os municípios que aderiram, em conjunto com a seleção de outros não aderentes, mas que tivessem características bastante similares às dos participantes do projeto. Tal procedimento teria facilitado a realização dos testes de causalidade (impacto) multivariados. Isso porque haveria um conjunto de informações que talvez dispensasse a inclusão de variáveis de controle, visto que se teria um pareamento previamente feito e razoavelmente bem equilibrado. Talvez o mais sensato e viável fosse implementar o projeto de forma experimental ou como piloto e realizar uma avaliação de impacto para alguns poucos municípios previamente selecionados em função de suas similaridades, determinando aleatoriamente que alguns fariam parte do projeto e outros não. Essa estratégia permitiria um desenho de avaliação de impacto bastante rigoroso e com caráter experimental ${ }^{11}$.

Conclui-se, com este estudo, que a simples adoção (ou adesão) não garante o êxito da política, pois devem ser levadas em conta as práticas concretas adotadas pelas organizações e os resultados produzidos localmente, no âmbito da "microimplementação" (BERMAN, 2007), cuja análise não faz parte dos objetivos da presente pesquisa.

9 Dado que esse projeto não foi desenhado para ser avaliado.

10 Tal procedimento é conhecido na literatura como avaliação ex-ante, a qual é realizada no começo de um programa, com o fito de dar suporte à decisão de implementar ou não o programa e ordenar os vários projetos segundo sua eficiência para alcançar os objetivos determinados. O elemento central da avaliação ex-ante é o diagnóstico, que auxilia na alocação dos recursos disponíveis de acordo com os objetivos propostos.

11 Este desenho é muito mais usado nas ciências naturais e se constitui num delineamento de pesquisa, no qual o pesquisador deseja verificar uma relação de causa e efeito, em que $X$ seria um tratamento qualquer e $Y$ o seu efeito. Para tanto, o pesquisador seleciona aleatoriamente elementos que receberão o tratamento e outros que não receberão. Nas ciências humanas, pode-se trabalhar com a mesma lógica, porque, normalmente, não há como selecionar aleatoriamente aqueles que recebem algum tratamento e os que não recebem, pois isso já deve ser dado naturalmente. 
ALMEIDA, M. R.; REBELATO, D. A. N. 0 inventário dos modelos de avaliação para políticas públicas. 2003. Disponível em: <http://sartori. orgfree.com/docs/modelo_de_politica_pub_ Mariana_Almeida.pdf>. Acesso em: 20 jul. 2013. ANGRIST, J.; KRUEGER, A. Empirical strategies in labor economics. In.: ASHENFELTER, O.; CARD, D. (Eds.). The handbook of labor economics, v. 3A, Chapter 23. Amsterdam: North-Holland, 1999. (Handbooks in Economics, n. 5).

BERMAN, Paul. El estudio de la macro y micro-implementación. In: VILLANUEVA, Luis F. Aguilar. La implementación de las políticas. México: Miguel Ángel Porrúa, 2007. p. 281-317.

CANO, Ignácio. Introdução à avaliação de programas sociais. 3. ed. Rio de janeiro: Editora FGV; 2006.

CARVALHO, Sonia Nahas de. Avaliação de programas sociais: balanço das experiências e contribuição para o debate. São Paulo em Perspectiva, São Paulo, v. 17, n. 3-4, p. 185-197, jul./dez. 2003.

COSTA, Frederico Lustosa da; CASTANHAR, José Cezar. Avaliação de programas públicos: desafios conceituais e metodológicos. Revista de Administração Pública, Rio de Janeiro, v. 37, n. 5, p. 962-969, set./out. 2003.

FARIA, Carlos Aurélio Pimenta de. A política da avaliação de políticas públicas. Rev. bras. Ci. Soc., v. 20, n. 59, p. 97-110, out. 2005.

FERREIRA, Marcos ArtêmioFischborn. Políticas Públicas. Material didático disponibilizado na disciplina Políticas Públicas e Desenvolvimento Regional do Programa de Pó-Graduação em Desenvolvimento Regional da Universidade de Santa Cruz do Sul. 2007. Arquivo formato Power-point.
GARCIA, Ronaldo Coutinho. Subsídios para organizar avaliações da ação governamental. Planejamento e Políticas Públicas, Brasília, n. 23 , p. 7-70, jan./jun. 2001.

HÖFLING, ELOISA DE MATTOS. Estado e Políticas (Públicas) Sociais. Cadernos Cedes, v. 21, n. 55 , novembro/2001.

REIS, Elisa P. Reflexões leigas para a formulação de uma agenda de pesquisa em políticas públicas. RBCS, v. 18, n. 51, p. 11-14, fev. 2003.

RAMOS, Marília Patta. Avaliação de Políticas e Programas Sociais: aspectos conceituais e metodológicos. IPEA, 2009. (Planejamento e Políticas Públicas, n. 32).

SOUZA, Celina. Políticas públicas: uma revisão da literatura. Revista Sociologias, Porto Alegre, n. 16, p. 20-45, jul/dez 2006.

STOTZ, Eduardo Navarro; ARAUJO, José Wellington Gomes. Promoção da saúde e cultura política: a reconstrução do consenso. Saude soc., v.13, n. 2, p. 5-19, 2004. ISSN 01041290.

VIANA, Ana Luiza. Abordagens metodológicas em políticas públicas. Revista de Administração Pública (RAP), Rio de Janeiro, v. 30, n.2, p. 5-43, mar./abr. 1996.

WILDAVSKY, A. Speaking truth to power: the art and craft of policy analysis. Boston, 1979.

WONG, L. R. e CARVALHO, J. A. The rapid process of aging in Brazil: serious challenges for Public policies. Brazilian Population Studies Journal, v. 23, n. 1, 2006.

Revista Brasileira de Monitoramento e Avaliação | Número 9 | Janeiro-Junho de 2015

Avaliação de impacto do Programa Inverno Gaúcho no estado do Rio Grande do Sul 